\title{
Influence of combustion on principal strain-rate transport in turbulent premixed flames
}

\author{
A. M. Steinberg \\ Institute for Aerospace Studies, University of Toronto, Toronto ON, Canada \\ B. R. Coriton, J. H. Frank \\ Combustion Research Facility, Sandia National Laboratories, Livermore CA, USA
}

\begin{abstract}
The transport of principal strain-rates $\left(s_{i}\right)$ was experimentally investigated using high-repetition-rate $(10 \mathrm{kHz})$ tomographic particle image velocimetry (T-PIV) and $\mathrm{OH}$ planar laser induced fluorescence (PLIF) in a $\mathrm{Re}_{\mathrm{j}}=$ 13, 000 turbulent premixed flame. These measurements allowed calculation of the source terms in the $s_{i}$ transport equation associated with the strain-rate and vorticity fields. Furthermore, the Lagrangian derivatives of $s_{i}$ could be calculated by tracking theoretical Lagrangian fluid particles through space and time using the T-PIV data. These Lagrangian derivatives and the resolved source terms allowed the combined effects of the unresolved source terms to be inferred, namely the pressure Hessian, viscous dissipation, density gradients, and viscosity gradients. Statistics conditioned on the location of the Lagrangian fluid particles relative to the flame showed slight reductions in the strain-rate and vorticity source-terms in the flame, indicating that these aspects of the turbulence were attenuated by the flame. Comparing the difference between the inferred source-terms in the vicinity of the flame to the non-reacting flow showed that attenuation of $s_{i}$ arose due to the combined effects of density and pressure gradients in the flame. The effects of flame-induced dilatation were small relative to the turbulent strain-rate and no change was found in the relative alignment of vorticity and strain-rate in the flame.
\end{abstract}

Keywords: Turbulent premixed flames, strain-rate, high-repetition-rate laser diagnostics, tomographic PIV, PLIF 


\section{Introduction}

The interaction between a premixed flame and the turbulent strain-rate field through which it propagates is a fundamental process in turbulent combustion. Such strain-rate/flame interactions alter the topology and scalar structure of the flame, as well as the magnitude and geometry of the turbulence [1-8]. As such, terms describing this interaction appear in various governing equations and must be modeled in simulations. These terms generally involve both the magnitude of the strain-rate field and its orientation relative to the flame surface, often taking the form $n_{i} n_{j} S_{i j}$, where $\hat{n}$ is the scalar gradient (flame surface) normal direction and $S_{i j}=$ $1 / 2\left(\partial u_{i} / \partial x_{j}+\partial u_{j} / \partial x_{i}\right)$ is the strain-rate tensor.

The strain-rate tensor can be expressed in its local eigenframe, wherein the strain-rates are represented by the three eigenvalues or principal strain-rates $\left(s_{i}\right.$, ordered $\left.s_{1}>s_{2}>s_{3}\right)$ that act in the directions of the corresponding eigenvectors $\left(\hat{e}_{i}\right)$. Strain-rate/flame interactions therefore typically are characterized by

$$
n_{i} n_{j} S_{i j}=s_{1}\left|\hat{e_{1}} \cdot \hat{n}\right|^{2}+s_{2}\left|\hat{e_{2}} \cdot \hat{n}\right|^{2}+s_{3}\left|\hat{e_{3}} \cdot \hat{n}\right|^{2}
$$

It therefore is necessary to understand how $\hat{e}_{i} \cdot \hat{n}$ and $s_{i}$ evolve through a flame.

Recent computational studies have elucidated several aspects of strain-rate/flame alignment. For example, Chakraborty et al. studied such alignment using 3D DNS of statistically planar flames with one-step chemistry at Reynolds numbers of about $\operatorname{Re}_{t}=u_{r}^{\prime} l / \nu \approx 50$ and over a range of Damköhler numbers $\left(\mathrm{Da}=\left(l / \delta_{l}\right) /\left(u_{r}^{\prime} / s_{l}\right)=\right.$ $0.3-6.8)$, and Karlovitz numbers $\left(\mathrm{Ka}=\delta_{l}^{2} / \lambda_{k}^{2}=9.8-34.3\right)$ [5]. Here, $l, \lambda_{k}$, and $u_{r}^{\prime}$ are the turbulence integral length scale, Kolmogorov length scale, and root-mean-squared velocity fluctuations in the reactants; $\delta_{l}$ and $s_{l}$ are the laminar flame (thermal) thickness and speed. Additionally, Hamlington et al. studied numerous aspects of turbulence-flame interaction using simulations of highly turbulent $\mathrm{H}_{2}$-air flames at $\mathrm{Da}=0.03-0.39$ and corresponding $\mathrm{Ka}=174.2-3.45$ [3]. At lower turbulence intensities, both studies found that dilatationrelated extensive strain-rate within the flame could dominate over the turbulent strain-rate. Hence, scalar gradients preferentially aligned with the eigenvector of the most extensive strain-rate, $\hat{e}_{1}$, within the flame. At higher turbulence-intensities, turbulence dominated over dilatation and the strain-rate/flame alignment resembled that found in passive scalar fields; scalar gradients aligned perpendicular to the most extensive strain-rate direction.

Regarding the principal strain-rate magnitudes, the relevant transport equations in a reacting flow can be written as 


$$
\begin{aligned}
\underbrace{\frac{\mathrm{D} s_{i}}{\mathrm{D} t}}_{L_{i}} & =\underbrace{-s_{i}^{2}}_{\tilde{T}_{1 i}}+\underbrace{\frac{1}{4}\left(\tilde{\omega}_{k} \tilde{\omega}_{k}-\tilde{\omega}_{i}^{2}\right)}_{\tilde{T}_{2 i}} \\
& +\underbrace{\nu \frac{\partial^{2} s_{i}}{\partial x_{k} \partial x_{k}}}_{\tilde{T}_{3 i}}-\frac{1}{\rho} \tilde{\Pi}_{i}+\tilde{T}_{4 i}+\tilde{T}_{5 i}
\end{aligned}
$$

where $\mathrm{D} / \mathrm{D} t=\partial / \partial t+u_{j} \partial / \partial x_{j}$ is the Lagrangian derivative, $\tilde{\omega}_{j}$ is the vorticity vector in the local strain-rate eigenframe $\left(\tilde{\omega}_{i}=E_{i j}^{T} \omega_{j}, E_{i j}^{T}\right.$ is the transpose (and hence inverse) of the orthogonal matrix $E=\left[\hat{e}_{1} \hat{e}_{2} \hat{e}_{3}\right]$ ), and $\tilde{\Pi}_{i}$ is the $i^{\text {th }}$ diagonal element of the pressure Hessian $\left(\Pi_{i j}=\partial^{2} p / \partial x_{i} \partial x_{j}\right)$ expressed in the strain eigenframe ( $\tilde{\Pi}=E^{T} \Pi E$ ). The terms $\tilde{T}_{1}-\tilde{T}_{3}$ and $\tilde{\Pi}_{i}$ take the same form in the non-reacting (constant density) transport equation, but are influenced by reaction through changes in the velocity, density, viscosity, and pressure fields. Furthermore, dilatation and the influence of heat release on the vorticity field fundamentally also affect the strainrate transport. The $\tilde{T}_{4}$ and $\tilde{T}_{5}$ terms in Eq. 2 arise due to density and viscosity gradients associated with combustion and take the form

$$
\begin{gathered}
\tilde{T}_{4}=E^{T}\left[\frac{1}{2 \rho^{2}}\left(\frac{\partial p}{\partial x_{i}} \frac{\partial \rho}{\partial x_{j}}+\frac{\partial p}{\partial x_{j}} \frac{\partial \rho}{\partial x_{i}}\right)\right] E \\
\tilde{T}_{5}=E^{T}\left[\frac{1}{2}\left(\frac{\partial \nu}{\partial x_{j}} \frac{\partial^{2} u_{i}}{\partial x_{k} \partial x_{k}}+\frac{\partial \nu}{\partial x_{i}} \frac{\partial^{2} u}{\partial x_{k} \partial x_{k}}\right)\right] E
\end{gathered}
$$

For non-reacting flows, Nomura and Post studied the mean contribution of the principal strain-rate source terms using DNS of decaying isotropic turbulence [9]. They classified these terms into local effects (i.e. $\tilde{T}_{1}, \tilde{T}_{2}$, and the locally induced portion of $\tilde{\Pi})$, non-local effects of $\tilde{\Pi}$, and viscous dissipation $\left(\tilde{T}_{3}\right)$. Local generation of the principal strain-rates typically was balanced by dissipation, with non-local effects being the smallest contribution.

In a reacting flow, Hamlington et al. studied the evolution of the vorticity across the flame [3]. They calculated budgets of the various source terms in the reacting vorticity transport equation and showed that those associated with the combustion (viz. dilatation and baroclinic torque) were significant at low turbulence intensities, but were dominated by turbulent production at higher intensities.

The insight gained from such computational studies can be enhanced and validated by experimental studies employing high-repetition-rate laser diagnostics. Such experiments allow investigation of more practicallyrelevant flames without the need for modeling, albeit with lower resolution, fewer detectable quantities, and potentially greater uncertainty. In this work, the evolution of the principal strain-rates are experimentally investigated using high-repetition-rate tomographic particle image velocimetry (T-PIV) and OH planar laser induced fluorescence (PLIF). These experiments allow explicit determination of the 3D principal strain-rates, their Lagrangian derivative, and several of the source terms in Eq. 2. 


\section{Experiment and Diagnostics}

The burner geometry employed was similar to that of the well-known Sandia Piloted Jet Flame series and is shown in Fig. 1 [10]. Premixed dimethyl-ether (DME) and air at $\phi=1.1\left(s_{l} \approx 0.4 \mathrm{~m} / \mathrm{s}, \delta_{l} \approx 0.6 \mathrm{~mm}\right)$ were provided to a central jet nozzle with diameter $D_{j}=7.45 \mathrm{~mm}$. This was surrounded by a pilot flame annulus $\left(\mathrm{CO}_{2} / \mathrm{N}_{2} / \mathrm{C}_{2} \mathrm{H}_{2} / \mathrm{H}_{2}\right.$ with air at $\left.\phi=0.6\right)$ and air co-flow with diameters of $18.2 \mathrm{~mm}$ and $254 \mathrm{~mm}$, respectively. All mass flow rates $(\dot{m})$ were metered using calibrated electromechanical flow controllers (MKS). The center of the measurement region was located at $(x / d, y / d, z / d)=(0,10,0)$, with the coordinate axes as shown in Fig. 1. Properties of the jet (subscript $j$ ), co-flow (subscript $c f$ ), and center of the measurement region are given in Table 1. The Damköhler and Karlovitz numbers place this flame in the thickened flamelet regime of turbulent premixed combustion, indicating that significant broadening of the preheat layer occurs. The Markstein length in this flame is expected to be near zero (effective Lewis number near unity), indicating that the effects of intrinsic flame instabilities are small [11].

Figure 1 also shows the $10 \mathrm{kHz}$ T-PIV and OH PLIF system. Tomographic PIV is well established for nonreacting flows and recently has been demonstrated in flames [12-14]. Complete details of the system used here, including a discussion of the measurement uncertainty, is provided in Ref. [14]. The T-PIV system consisted of a high-repetition-rate laser (Quantronix, Dual-Hawk-HP-240L) and four high-speed CMOS cameras (Vision Research Phantom v1610). The laser beams were formed into a collimated sheet-like volume, which was cropped with a rectangular slit aperture into a $3.45 \mathrm{~mm}$ thick laser volume that had less than $10 \%$ variation in fluence across the width. Laser light scattering from ca. $0.3 \mu \mathrm{m}$ aluminum oxide particles was simultaneously imaged onto the cameras, which were equipped with macro-lenses (Tamron, f.l. $=180 \mathrm{~mm}$ ) and Scheimpflug adapters. The time delay between pulses from each laser head was $8 \mu$ s. Frame straddling was employed, with the cameras operating at $20 \mathrm{kHz}$ and a resolution of $1024 \times 768 \mathrm{px}$.

Tomographic PIV processing consisted of camera volume calibration, particle image preprocessing, 3D particle location reconstruction, vector calculation, and post-processing, all performed using a commercial software package (LaVision DaVis 8.0). The volume calibration was performed using seven co-planar equidistant views of a 2D dotted transparent film target, and was refined using a volume self-calibration procedure. The 3D particle positions within the probe volume were reconstructed using four iterations of a Multiplicative Algebraic Reconstruction Tomography (MART) algorithm. The size of the reconstructed volume was $17.0 \times 11.5 \times 3.4 \mathrm{~mm}^{3}$, corresponding to $1133 \times 768 \times 228$ voxels. Inter-frame particle displacements were determined by performing multipass cross-correlation analysis using a final interrogation region size of $24 \times 24 \times 24$ voxels $\left(360 \times 360 \times 360 \mu \mathrm{m}^{3}\right)$ with a $75 \%$ overlap, yielding 919,296 vectors with a spacing of $90 \mu \mathrm{m}$. Smoothing of the velocity vectors was performed using a penalized least squares method that had the same $-3 \mathrm{~dB}$ cutoff frequency as a $4 \times 4 \times 4$ moving average filter [15]. Visual tracking indicated that the smallest spatial scale that could be consistently resolved was 
$\lambda_{m} \approx 0.7 \mathrm{~mm}$ (approx. 8 vector spaces, 2 interrogation boxes). It is noted that the smallest vortical structures, which were approximately $6 \lambda_{\mathrm{k}}=420 \mu \mathrm{m}$, could not quite be resolved.

PLIF imaging of $\mathrm{OH}$ at a repetition-rate of $10 \mathrm{kHz}$ was used to measure the flame topography. The second harmonic of a Nd:YAG-pumped dye laser (Edgewave IS6II and Sirah Credo) with $175 \mu \mathrm{J} /$ pulse was tuned to approximately $283.3 \mathrm{~nm}$ to excite the Q1(7) transition of the A-X(1,0) band of $\mathrm{OH}$. This laser beam was formed into a sheet and overlapped with the central plane of the $532 \mathrm{~nm}$ T-PIV laser volume using a dichroic mirror. Triggering of the PLIF laser system was configured such that each dye laser pulse was bracketed by the T-PIV pulses. The OH PLIF signal was recorded using a high speed intensified CMOS camera (Vision Research, Phantom V7.3). The OH PLIF images were not corrected for beam profile variations, but were normalized by the peak LIF signal within each image to account for shot-to-shot fluctuations in the laser energy. Registration of the OH PLIF measurement plane within the T-PIV probe volume was performed using a similar target as for the T-PIV calibration, but with a larger and coarser grid pattern [14].

The adequacy of the temporal resolution to measure the time-scale of smallest resolved turbulence length-scale can be described by the ratio of the inter-measurement time $\left(\Delta t_{\mathrm{m}}\right)$ and the turn-over time of the smallest resolved turbulent structures $\left(\tau\left(\lambda_{\mathrm{m}}\right)\right)$, viz. $C_{e}=\Delta t_{\mathrm{m}} / \tau\left(\lambda_{\mathrm{m}}\right)$ should be less than $1 / 2$ to satisfy the Nyquist criterion. From standard turbulence theory, the time-scale associated with any length-scale, $\lambda$, is $\tau(\lambda)=\left(\lambda^{2} / \epsilon\right)^{1 / 3}$ and $\epsilon=u^{\prime 3} / \delta$. Hence,

$$
C_{\mathrm{e}}=\frac{u^{\prime} \Delta t_{\mathrm{m}}}{\lambda_{\mathrm{m}}^{2 / 3} \delta^{1 / 3}}=\frac{\nu \operatorname{Re}_{\mathrm{t}} \Delta t_{\mathrm{m}}}{\lambda_{\mathrm{m}}^{2 / 3} \delta^{4 / 3}}
$$

In the current experiment, $\Delta t_{\mathrm{m}}=0.1 \mathrm{~ms}$ and $\lambda_{\mathrm{m}}=0.72 \mathrm{~mm}$, resulting in $C_{e}=0.15$. Hence, the measurements were well-resolved in time.

\section{Data analysis methods}

\subsection{Lagrangian Derivatives}

The Lagrangian derivatives required for this analysis could theoretically be calculated directly from the measured Eulerian velocity fields. However, this method requires calculating the spatial and temporal gradient of $s_{i}$, which is itself based on spatial velocity gradients. Spatial gradients in PIV, and particularly T-PIV, tend to exhibit noise. It therefore was found that calculating $\mathrm{D} s_{i} / \mathrm{D} t$ from Eulerian derivatives was not sufficiently accurate.

The Lagrangian derivatives therefore were calculated by following theoretical Lagrangian fluid particles (LFP) in space and time. That is, the Lagrangian derivative of any property can be calculated by following a theoretical fluid particle, the motion of which is dictated by the Lagrangian velocity field $(\vec{U})$. The position of such an LFP

at time $t^{*}$ is $\left.\vec{X}\right|_{t^{*}}=\vec{X}\left(\vec{X}_{0}, t_{0} \mid t^{*}\right)$, where $\vec{X}_{0}$ was the position of the LFP at $t_{0}$. The LFP position evolves according to

$$
\left.\frac{\mathrm{d} \vec{X}}{\mathrm{~d} t}\right|_{t^{*}}=\left.\vec{U}\right|_{t^{*}} \quad \text { where }\left.\quad \vec{U}\right|_{t^{*}}=\vec{u}\left(\left.\vec{X}\right|_{t^{*}}, t^{*}\right)
$$


The Lagrangian derivative of $s_{i}$ can then be calculated from a finite difference method and the values of $s_{i}$ associated with a given LFP at subsequent times. A second-order central difference method was used here. This method does not require calculating the gradients of $s_{i}$.

Several previous DNS investigations have been performed using theoretical Lagrangian fluid particles to study turbulence and turbulent combustion; a more complete description of Lagrangian tracking can be found in the literature [16-18]. Recently, Yang et al. implemented a backward-particle-tracking method to study the non-local geometry of Lagrangian structures in isotropic turbulence [16]. It was found that backward tracking increases robustness, prevents exponential dispersion, and better preserves required quantities than forward tracking.

Backward-tracking therefore was implemented in the current T-PIV data set. That is, LFPs were computationally placed into the experimentally determined flow fields on a uniform grid at the time of the last measurement. The initial grid was equivalent to the T-PIV grid, resulting in LFPs every $90 \mu \mathrm{m}$ in each direction. The LFP trajectories were then tracked backwards using Eq. 6 until the time that they entered the measurement volume $\left(t_{0}\right.$ for that LFP). As each LFP left the measurement volume, a new particle was inserted at the top of the domain in order to keep the total number of particles constant. Four interpolated time steps were used between each measured time step, and four-dimensional (nominally fourth-order) cubic-spline interpolation (in both space and time) was used to calculate the velocity at the instantaneous particle locations [19].

The Lagrangian derivatives thus calculated are sensitive to the accuracy with which the LFPs are tracked. This was assessed by comparing the directly-measured T-PIV particle fields around subsequent locations of a given LFP. If the LFP was tracked correctly, the T-PIV particle fields around the LFP should be similar. Cross-correlations therefore were performed between the particle fields in $32 \times 32 \times 32$ voxel regions around subsequent location of 200,000 LFPs. Figure 2 shows the PDFs of the correlation peak location in pixels for each coordinate direction. As can be seen, the most probable correlation-peak was at zero-shift. The mean magnitude of the correlation peak shift in each direction was approximately 10 voxels, or $150 \mu \mathrm{m}$. The LFPs therefore appear to be well tracked through time. However, to add robustness to the subsequent analysis, the turbulence properties associated with any LFP were taken to be the average at the four nearest velocity vector locations on the T-PIV grid. Considering the resolution of the measurements, this has largely a de-noising effect. It is noted that tracking LFPs directly from the T-PIV particles was not possible due to the seed density (it was not possible to distinguish individual T-PIV particles through time).

\subsection{Flame Identification}

The flame topology in the $z=0$ plane was identified from the gradient of the OH PLIF signal using the algorithm described in Ref. [20]. Rapid OH generation in premixed flames occurs at temperatures exceeding about $1500 \mathrm{~K}$. Hence, this contour represents a location towards the products side of the flame. Each flame contour was mathematically defined as a parametric space curve, $\vec{F}(\xi, t)=x_{f}(\xi, t) \hat{i}+y_{f}(\xi, t) \hat{j}$, where $\xi$ is a 
coordinate along the flame surface.

Due to the planar nature of the flame measurements, Lagrangian fluid particles were only considered if they remained within $z= \pm 180 \mu \mathrm{m}$ (one interrogation box) of the PLIF plane for a minimum of the three consecutive measurements times required to compute the Lagrangian derivatives $(0.2 \mathrm{~ms})$. This restriction did not remove many LFPs from consideration, since mean $z$-velocity was nearly zero. The instantaneous planar distance of a LFP from the flame was then calculated as

$$
D_{f}\left(\left.\vec{X}\right|_{t^{*}}\right)=\min \left(\left|\vec{X}_{2 D}\right|_{t^{*}}-\vec{F}\left(\xi, t^{*}\right) \mid\right)
$$

where $\vec{X}_{2 D}$ represents only the $x$ - and $y$-components of $\vec{X}$.

\subsection{Particle conditioning}

Due to the highly turbulent nature of the flame and the inability to measure the local flame scalar structure (i.e. instantaneous progress variable field), LFPs were classified by whether they were 'in the flame' or 'away from the flame'. Figure 3 shows profiles of various significant properties through a $\phi=1.1$ laminar DME/air flame computed using Cantera. LFPs deemed 'in the flame' at a given time were those that were within $0 \leq D_{f} \leq 1 \mathrm{~mm}$ (i.e. in the flame preheat layer), whereas those deemed 'away from the flame' were at $D_{f} \geq 3 \mathrm{~mm}$ (not shown in Fig. 3). These two scenarios will be denoted as $(\cdot)_{f}$ and $(\cdot)_{n r}$ (non-reacting), respectively. The region considered to be in the flame for statistical conditioning is shown in Fig. 3. In the range of $1 \leq D_{f} \leq 3 \mathrm{~mm}$, local preheat layer broadening may determine whether the LFP is influenced by the flame.

\subsection{Summary of Analysis}

The overall analysis followed the following steps:

1. At each instant in time, the flame surface topology was calculated from the OH PLIF gradient

2. From the velocity field measurements, Lagrangian fluid particle trajectories were calculated through backward particle tracking.

3. Of these, particles were selected that remained within $z= \pm 180 \mu \mathrm{m}$ for a minimum of $0.2 \mathrm{~ms}$ ( 3 measurement times) and were conditioned based on their distance to the flame.

4. At each particle location and time, the following properties were calculated.

(a) principal strain-rates, $s_{i}$

(b) Lagrangian derivative of principal strain-rates, $L_{i}$

(c) Vorticity field in principal strain eigenframe: $\tilde{\omega}_{i}$

(d) principal strain-rate source terms: $\tilde{T}_{1}-\tilde{T}_{2}$ 
(e) Distance to the nearest flame surface, $D_{f}$

The viscous term in Eq. 2 was not directly calculated due to the difficulty in calculating third-derivatives of the velocity field and the unknown local viscosity within the flame. However,

$$
\tilde{T}_{i}^{*}=\tilde{\Pi}_{i}+\tilde{T}_{3 i}+\tilde{T}_{4 i}+\tilde{T}_{5 i}=L_{i}-\tilde{T}_{1 i}-\tilde{T}_{2 i}
$$

was calculated.

In total, approximately $4 \times 10^{6}$ LFPs were used for this analysis, which were compiled from 2500 individual measurements. However, not all LFPs were statistically independent due to the velocity field over-sampling. It is estimated that approximately $1 \times 10^{5}$ independent LFPs were used. Estimates of the uncertainty in the velocity gradients were made from the pseudo-laminar regions of the co-flow. These indicated random noise levels with magnitudes in the range of $1000 \mathrm{~s}^{-1}$ for the strain-rate components, but occasionally reaching $2000 \mathrm{~s}^{-1}$. Considering the large number of samples used for the presented statistics, and that the mean measured strainrate magnitude was approximately $10000 \mathrm{~s}^{-1}$, this level of uncertainty is not expected to significantly affect the conclusions.

\section{Results}

\subsection{Lagrangian Derivatives}

Figure 4 shows the position of a small number of LFPs in the vicinity of the flame and the $\mathrm{OH}$ field over three time steps. It can be observed that the flame slowly overtakes the LFPs due to its propagation speed. Figure 5 shows the PDFs of $\left.L_{1}\right|_{f}$ and $\left.L_{1}\right|_{n r}$, which are typical for all $L_{i}$. As can be seen, all Lagrangian derivatives had lower magnitude for LFPs inside the flame.

\subsection{Straining Terms, $\tilde{T}_{1 i}$}

Figure 6 shows the conditional PDFs of $\tilde{T}_{1 i}$, showing that $\left|\tilde{T}_{11}\right|$ decreased significantly for Lagrangian fluid particles in the flame; little change occurred in $\left|\tilde{T}_{12}\right|$ and $\left|\tilde{T}_{13}\right|$. This result is interesting since one may intuitively expect flame-related dilatation, which results in an extensive strain-rate, to increase $\left.s_{1}\right|_{f}$ and hence $\left|\tilde{T}_{11}\right|_{f}$. The observed results indicate that dilatation is relatively insignificant compared to the turbulent strain-rate and other source-terms, which is consistent with simulations of highly-turbulent flames [3,5].

Simulation of a freely propagating laminar $\phi=1.1$ DME-air flame using Cantera indicates that the maximum combustion-generated velocity increase over a $720 \mu \mathrm{m}$ region (two interrogation volumes) would result in a dilatation-related strain-rate of approximately $3000 \mathrm{~s}^{-1}$, whereas the mean turbulent strain-rate magnitude in this system was measured to be approximately $\left(s_{i} s_{i}\right)^{1 / 2}=10000 \mathrm{~s}^{-1}$. Hence, turbulent straining appears to dominate over dilatation in this flame. The observed decrease in $\left|\tilde{T}_{1 i}\right|$ in the flame therefore is associated with increased 
attenuation of the turbulence due to the other source terms in Eq. 2.

\subsection{Vorticity Terms, $\tilde{T}_{2 i}$}

Figure 7 shows the conditional PDFs of $\tilde{T}_{21}$, which are typical for all $\tilde{T}_{2 i}$. These once again show a slight decrease in the flame region. The $\tilde{T}_{2 i}$ terms are indicative of the vorticity vector magnitude and orientation with respect to the principal strain-rate field. That is, the alignment of the vorticity vector with the principal strain-rate eigenvectors can be expressed as

$$
\theta_{i}=\left|\hat{e}_{i} \cdot \hat{\omega}\right|=\tilde{\omega}_{i} /\left(\tilde{\omega}_{k} \tilde{\omega}_{k}\right)^{1 / 2}
$$

where $\hat{\omega}$ is a unit vector in the $\vec{\omega}$ direction. Preferential alignment of the vorticity with a particular strain-rate axis is indicated by values of $\theta_{i}$ close to unity. In non-reacting isotropic turbulence, it is known that $\hat{\omega}$ preferentially aligns with $\hat{e}_{2}$, misaligns with $\hat{e}_{3}$, and randomly aligns with $\hat{e}_{1}$. Hamlington et al. and Gamba et al. found that combustion did not significantly change in vorticity/strain-rate alignment in their reacting flows [3, 21]

Figure 8 shows conditional PDFs of $\theta_{i}$, demonstrating the measured preferential alignment of vorticity with the strain-rate eigenvectors. The vorticity vector was preferentially aligned with $\hat{e}_{2}$ and misaligned with both $\hat{e}_{1}$ and $\hat{e}_{3}$, exhibiting stronger misalignment with the latter. None of these alignment characteristics changed for Lagrangian particles in the flame. Hence, the reduction in $\left.\tilde{T}_{2 i}\right|_{f}$ relative to $\left.\tilde{T}_{2 i}\right|_{n r}$ is due to attenuation of vorticity in the reactants in the flame caused by a combination of dilatation and baroclinic torque effects [3, 22, 23].

\subsection{Remaining terms, $\tilde{T}_{i}^{*}$}

The remaining terms in Eq. 2 could not be explicitly determined by these measurements, but can be inferred

using Eq. 8. Away from the flame, $\tilde{T}_{i}^{*}$ is equivalent to the sum of the viscous term, $\tilde{T}_{3}$, and the pressure Hessian, $\tilde{\Pi}_{i}$. For Lagrangian particles in the flame, it accounts for $\tilde{T}_{3}, \tilde{\Pi}_{i}$, and terms arising due to density and viscosity gradients. The conditional PDFs of $\tilde{T}_{i}^{*}$ are shown in Fig. 9 . As can be seen, $\tilde{T}_{1}^{*}$ and $\tilde{T}_{3}^{*}$ are preferentially positive, whereas $\tilde{T}_{2}^{*}$ has a nearly symmetric PDF. Assuming that viscous dissipation is relatively small over the relevant time scales, the $\left.\tilde{T}_{i}^{*}\right|_{n r}$ curves essentially represent the diagonal elements of the pressure Hessian in the strainrate eigenframe. Indeed, these measured PDFs are qualitatively very similar to the $\tilde{\Pi}_{i}$ PDFs computed in the non-reacting DNS of Nomura and Post [9].

For all strain-rate components, the most probable $\tilde{T}_{i, f}^{*}$ and $\tilde{T}_{i, n r}^{*}$ was unaffected by the flame. However, the widths of the PDFs for all three strain components were narrower for LFPs near the flame, indicating an overall reduction in these terms near the flame. This reduction can be attributed to the effects of the local flame on the pressure Hessian or the effects of the density- and viscosity-gradient related terms $\left(\tilde{T}_{4 i}\right.$ and $\left.\tilde{T}_{5 i}\right)$ becoming active within the flame.

The influence of the flame on the pressure Hessian-term can be estimated from a 1D laminar flame calculation 


$$
\frac{1}{\rho_{u}} \frac{\partial^{2} p}{\partial x^{2}} \approx \frac{1}{\rho_{u}} \frac{1}{\delta_{l}} \frac{\partial p}{\partial x} \approx \frac{1}{\rho_{u}} \frac{1}{\delta_{l}} \frac{\rho_{u} \tau s_{l}^{2}}{\delta_{l}} \approx 5 \times 10^{6} \mathrm{~s}^{-2}
$$

Comparing this value to those in Fig. 9 indicates that the influence of the flame on the pressure Hessian is not the dominant effect. Furthermore, an order of magnitude analysis similar to that of Mueller et al. indicates that $\tilde{T}_{5 i}$ is

small relative to $\tilde{T}_{4 i}$ and the main mechanism attenuating principal strain-rates in the reactants is combined density and pressure gradients $\left(\tilde{T}_{4 i}\right)$ [22]. This leads to lower values of $s_{i}$, and hence $\tilde{T}_{1 i}$ for Lagrangian fluid particles the flame. The reduction is greatest for $\tilde{T}_{1}^{*}$, followed by $\tilde{T}_{3}^{*}$ and $\tilde{T}_{2}^{*}$, which is consistent with the reductions in $\tilde{T}_{1 i}$.

\section{Conclusions}

This work utilized tracking of Lagrangian fluid particles through a 4D experimental data set to study the source-terms in the turbulent strain-rate transport equation for reacting flows. The strain-rate-related source terms $\left|\tilde{T}_{1 i}\right|$ (and hence $\left|s_{i}\right|$ ) were lower for LFPs in the flame than for those in non-reacting regions of the flow, indicating that combustion acts to attenuate the turbulent strain-rate. Similarly, the vorticity-related source terms $\left(\tilde{T}_{2 i}\right)$ had lower magnitude for LFPs in the flame due to the flame attenuating vorticity in the reactants, which is consistent with previous studies of vorticity transport. The main mechanism of strain-rate attenuation within the flame was found to be the source term associated with combined pressure and density gradients. This effect was most apparent in $\tilde{T}_{1}^{*}$, followed by $\tilde{T}_{3}^{*}$ and $\tilde{T}_{2}^{*}$, which is consistent with the relative reductions in $\tilde{T}_{1 i}$. The experimental results are consistent with DNS studies that indicate the influence of heat release on turbulence becomes increasingly insignificant at high turbulence intensities. Future work will investigate the influence of the local and global turbulence parameters (e.g. Damköhler and Karlovitz numbers) on the observed phenomena.

\section{Acknowledgements}

A. Steinberg acknowledges the support of the US Air Force Office of Scientific Research under Grant No. FA9550-13-1-0070, Project Monitor Dr. Chiping Li, and NSERC under Grant No. RGPIN 413232. The authors acknowledge the support of the US Department of Energy, Office of Basic Energy Sciences, Division of Chemical Sciences, Geosciences, and Biosciences. Sandia National Laboratories is a multiprogram laboratory operated by Sandia Corporation, a Lockheed Martin Company, for the US Department of Energy under contract DE-AC0494-AL85000. The technical support of Mr. Erxiong Huang also was greatly appreciated.

\section{References}

[1] B. Coriton, J. H. Frank, A. Gomez, Combust. Flame 160 (2013) 2442-2456.

[2] A. M. Steinberg, J. F. Driscoll, Combust. Flame 156 (2009) 2285-2306.

[3] P. E. Hamlington, A. Y. Poludnenko, E. S. Oran, Phys. Fluids 23 (2011) 125111.

[4] N. Chakraborty, N. Swaminathan, Combust. Sci. Technol. 185 (2013) 676-709. 
[5] N. Chakraborty, N. Swaminathan, Phys. Fluids 19 (2007) 045104.

[6] M. Shimura, T. Ueda, G. M. Choi, M. Tanahashi, T. Miyauchi, Proc. Combust. Inst. 33 (2011) 775-782.

[7] G. Hartung, J. Hult, C. Kaminski, J. Rogerson, N. Swaminathan, Phys. Fluids 20 (2008) 035110.

[8] S. H. Kim, H. Pitsch, Phys. Fluids 19 (2007) 115104.

[9] K. K. Nomura, G. K. Post, J. Fluid Mech. 377 (1998) 65-97.

[10] R. S. Barlow, J. H. Frank, Proc. Combust. Inst. 27 (1998) 1087-1095.

[11] X. Qin, Y. Ju, Proc. Comb. Inst. 30 (2005) 233-240.

[12] J. Westerweel, G. E. Elsinga, R. J. Adrian, Annu. Rev. Fluid Mech. 45 (2013) 409-436.

[13] J. Weinkauff, D. Michaelis, A. Dreizler, B. Böhm, Exp. Fluids 54 (2013) 1624.

[14] B. Coriton, A. M. Steinberg, J. H. Frank, Exp. Fluids 55 (2014) 1743.

[15] D. Garcia, Exp. Fluids 50 (2011) 1247-1259.

[16] Y. Yang, D. I. Pullin, I. Bermejo-Moreno, J. Fluid Mech. 654 (2010) 233-270.

[17] C. Meneveau, Annu. Rev. Fluid Mech. 43 (2011) 219-245.

[18] P. K. Yeung, Annu. Rev. Fluid Mech. 34 (2002) 115-142.

[19] P. K. Yeung, S. B. Pope, J. Comput. Phys. 79 (1998) 373-416.

[20] A. M. Steinberg, I. Boxx, M. Stöhr, C. D. Carter, W. Meier, Combust. Flame 157 (2010) 2250-2266.

[21] M. Gamba, N. T. Clemens, O. A. Ezekoye, Meas. Sci. Technol. 24 (2013) 024003.

[22] C. J. Mueller, J. F. Driscoll, D. L. Reuss, M. C. Drake, M. E. Rosalik, Combust. Flame 112 (3) (1998) $342-358$.

[23] D. S. Louch, K. N. C. Bray, Combust. Flame 125 (4) (2001) 1279-1309. 
7. *

Table 1: Experimental conditions.

\begin{tabular}{lc|lc}
\hline \multicolumn{2}{c|}{ Jet } & \multicolumn{2}{c}{ Measurement } \\
\multicolumn{2}{c}{ exit } & & \multicolumn{2}{c}{ location } \\
\hline$\dot{m}_{\text {air }}$ & $1.0 \mathrm{~g} / \mathrm{s}$ & $l$ & $3.1 \mathrm{~mm}$ \\
$\dot{m}_{\mathrm{DME}}$ & $0.13 \mathrm{~g} / \mathrm{s}$ & $u_{r}^{\prime}$ & $1.7 \mathrm{~m} / \mathrm{s}$ \\
$\bar{u}_{\mathrm{j}}$ & $21 \mathrm{~m} / \mathrm{s}$ & $\mathrm{Re}_{t}$ & $0.4 \times 10^{3}$ \\
$\bar{u}_{\mathrm{cf}}$ & $0.30 \mathrm{~m} / \mathrm{s}$ & $\lambda_{k}$ & $70 \mu \mathrm{m}$ \\
$\operatorname{Re}_{\mathrm{j}}\left(\bar{u}_{\mathrm{j}} D_{\mathrm{j}} / \nu_{\mathrm{j}}\right)$ & $13 \times 10^{3}$ & $\mathrm{Da}$ & 1.3 \\
$\tau=\rho_{u} / \rho_{b}-1$ & 7.4 & $\mathrm{Ka}$ & 60 \\
\hline
\end{tabular}


8. *

Figure Captions

Fig. 1. Experimental setup for $10 \mathrm{kHz}$ T-PIV/OH PLIF system.

Fig. 2. PDFs of cross-correlation-peak shift between T-PIV particle fields around Lagrangian particles at subsequent times.

Fig. 3. Profiles of normalized temperature, density, velocity and $\mathrm{OH}$ concentration from laminar flame simulation.

Fig. 4. Example of tracking a few Lagrangian fluid particles as they interact with the flame in a subvolume of the T-PIV measurement volume. Dimensions are in millimeters.

Fig. 5. Conditional PDFs of the Lagrangian derivative of the first principal strain-rate, $L_{1}$.

Fig. 6. Probability distribution functions of strain source-terms $\left(\tilde{T}_{1 i}\right)$ conditioned on LFPs near the flame and away from the flame.

Fig. 7. Conditional PDFs of $\tilde{T}_{21}$, which are typical of $\tilde{T}_{2 i}$.

Fig. 8. Conditional PDFs of the alignment between the vorticity vector and the principal strain-rate eigenvectors near and far from the flame.

Fig. 9. Conditional PDFs of $\tilde{T}_{i}^{*}$ showing the contribution of the unmeasured terms in Eq. 2. 